\title{
Hemochromatosis Mistakenly Treated as Rheumatoid Arthritis
}

\author{
Gürkan AKGÖL, ${ }^{1}$ Hasan ULUSOY, ${ }^{2}$ Ayhan KAMANLI ${ }^{1}$ \\ ${ }^{1}$ Division of Rheumatology, Department of Physical Medicine and Rehabilitation, Medical Faculty of Firat University, Elaziğ, Turkey \\ ${ }^{2}$ Department of Rheumatology, Erzurum Regional Training and Research Hospital, Erzurum, Turkey
}

\begin{abstract}
A 44-year-old female patient was admitted to our clinic with complaints of episodic pain, swelling attacks, and progressive deformity in both wrists and metacarpophalangeal joints lasting for five years. Based on her complaints, she had been diagnosed with rheumatoid arthritis and taken methotrexate, sulphasalazine, and prednisolone for two years, however, the patient had discontinued her treatment a year earlier due to ongoing symptoms. Physical examination revealed limited range of motion of both wrists and flexion deformity of the fingers without active inflammation signs. Hand X-ray showed typical signs of osteoarthritis characterized by narrowed joint spaces, subchondral sclerosis, and cyst formation. The distal and proximal interphalangeal joints were markedly preserved, and there were large, hook-like osteophytes in the heads of the metacarpal bones, indicating a typical presentation of hemochromatosis. Laboratory tests revealed that the erythrocyte sedimentation rate and C-reactive protein and uric acid levels were within the normal range, and the antibody tests were negative for rheumatoid factor, anti-nuclear antibodies, and anti-cyclic citrullinated peptides. Although the serum iron and ferritin levels were normal, there was a high transferrin saturation rate. Magnetic resonance imaging demonstrated a hepatic iron concentration of 44 $\mu \mathrm{mol} / \mathrm{g}$ (reference: $<36 \mu \mathrm{mol} / \mathrm{g}$ ). Genetic studies showed homozygous for the H63D mutation. Based on these findings, the patient was diagnosed with hereditary hemochromatosis and scheduled for follow-up visits. In conclusion, hemochromatosis should be considered in the differential diagnosis in patients suffering from slow-progressing arthritis with chronic deformity.

Keywords: Hemochromatosis; osteoarthritis; radiology; rheumatoid arthritis.
\end{abstract}

Hereditary hemochromatosis $(\mathrm{HH})$ is a common autosomal recessive disease that affects Caucasian populations at a prevalence rate ranging from one in 200 to one in $500 .^{1}$ Increased intestinal absorption and the visceral deposition of iron can lead to the phenotypic features of hepatic cirrhosis, cardiomyopathy, diabetes mellitus (DM), pituitary dysfunction, Sicca syndrome (Sjogren's syndrome), and skin pigmentation. ${ }^{2}$ Chronic progressive arthritis, predominantly affecting the second and third metacarpophalangeal (MCP) joints, proximal interphalangeal (PIP) joints, and wrists, is the presenting complaint in approximately half of the cases of HH. ${ }^{1-3}$ Therefore, it is often confused with other rheumatic diseases that affect the hand joints, especially rheumatoid arthritis (RA), generalized osteoarthritis (GOA), psoriatic arthritis (PsA), and gouty arthritis. In this report, we present the case of a patient with $\mathrm{HH}$ who was misdiagnosed and treated for RA.

\section{CASE REPORT}

A 44-year-old female patient was referred to our rheumatology clinic with complaints of pain and morning stiffness that had lasted for less than 30 minutes along with recurrent episodes of swelling and progressive deformities involving both wrists and the MCP joints that had begun five years earlier. In addition, she had suffered for the previous two years from gradually increasing pain and restricted range of motion (ROM) in her hip joints, which led to difficulty in walking. The patient had been diagnosed with RA in another rheumatology clinic and took methotrexate

Received: October 23, 2012 Accepted: January 22, 2013

Correspondence: Hasan Ulusoy, M.D. Fırat Üniversitesi Tıp Fakültesi, Fiziksel Tıp ve Rehabilitasyon Anabilim Dalı, Romatoloji Bilim Dalı, 23119 Elazığ, Turkey. Tel: +90424 - 2478928 e-mail: ulusoyh@mynet.com 
(15 mg/week), sulfasalazine (2 g/day), and prednisone $(7.5 \mathrm{mg} /$ day $)$ for two years. With this therapy, her complaints had only decreased minimally, and she discontinued this therapy one year prior to her admission at our facility because of persistent pain and progressive deformities.

A physical examination revealed decreased $\mathrm{ROM}$ in both wrists and the MCP joints with accompanying flexion deformities; however, the affected joints did not demonstrate any signs of active inflammation. Furthermore, the patient's hip joint movements were painful and restricted. Typical signs of osteoarthritis (OA) (irregular narrowing of joint spaces, subchondral sclerosis, and sclerotic cyst formation) were seen on hand radiograms (Figure 1). Strikingly, the distal interphalangeal (DIP) and PIP joints were intact, and hook-like osteophytic formations on the heads of the metacarpal bones were seen, which is typical of hemochromatosis. Pelvic radiograms revealed irregular joint space narrowing, sclerotic changes, and subchondral cysts involving the hip joints (Figure 2). Additionally, the laboratory findings showed a normal erythrocyte sedimentation rate (ESR) and $\mathrm{C}$-reactive protein (CRP) levels, and the patient tested negative for rheumatoid factor (RF) as well as anti-cyclic citrullinated peptide (anti-CCP) and antinuclear antibodies (ANA). Furthermore, the serum uric acid level was

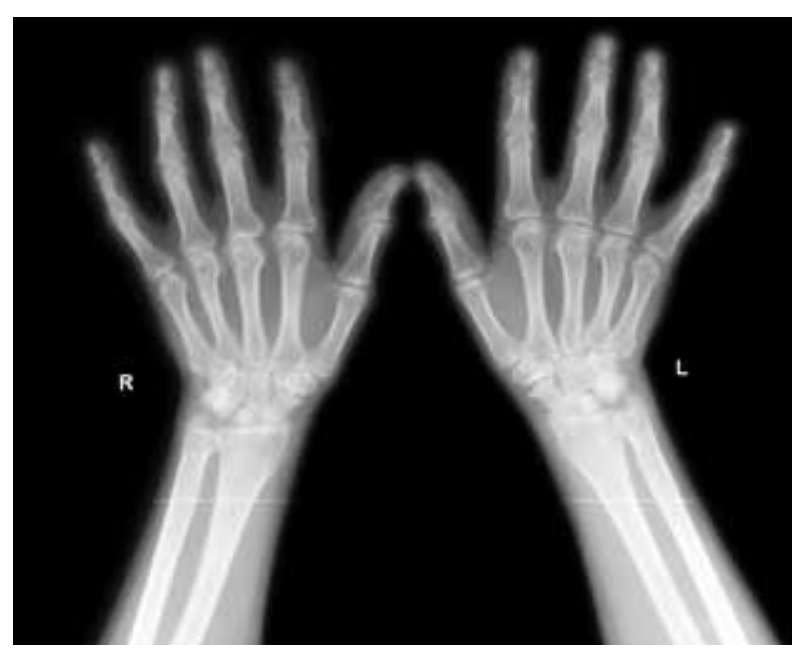

Figure 1. Marked loss of articular cartilage and subchondral sclerosis of the metacarpophalangeal joints is shown. Note the hook-like osteophytic formations on the heads of the metacarpal bones, which are typical of hemochromatosis. Remarkably, the distal interphalangeal and proximal interphalangeal joints are spared. normal, and the liver function test results were within normal limits. Although the serum iron and ferritin levels were also within normal limits, the transferrin saturation rate was higher (69.71\%) than normal $(<55 \%)$. Moreover, the patient's estimated hepatic iron concentration was $44 \pm 20 \mu \mathrm{mol} / \mathrm{g}$ (normal $<36 \mu \mathrm{mol} / \mathrm{g}$ ) using $\mathrm{T}_{2}$ sequences in abdominal magnetic resonance imaging (MRI). A genetic examination also determined the existence of a homozygous H63D mutation; therefore, the patient was diagnosed with $\mathrm{HH}$ based on the clinical, radiological, and laboratory findings.

\section{DISCUSSION}

The term hemochromatosis encompasses all diseases with iron accumulation disorders. Prolonged and excessive iron ingestion and repeated blood transfusions that take place with hypoproliferative anemia and thalassemia may result in secondary hemochromatosis. However, in most cases, a concomitant disease resulting in hemochromatosis cannot be found, so the disease is termed $\mathrm{HH} .{ }^{3}$ Most patients are usually diagnosed between the ages of 40 and 50, and in many investigations, $\mathrm{HH}$ has been observed between two and eight times more often in men, with the most frequently seen symptoms being asthenia, fatigue, arthralgia, abdominal pain, loss of libido, hepatomegaly, and cardiomyopathy. ${ }^{4-6}$

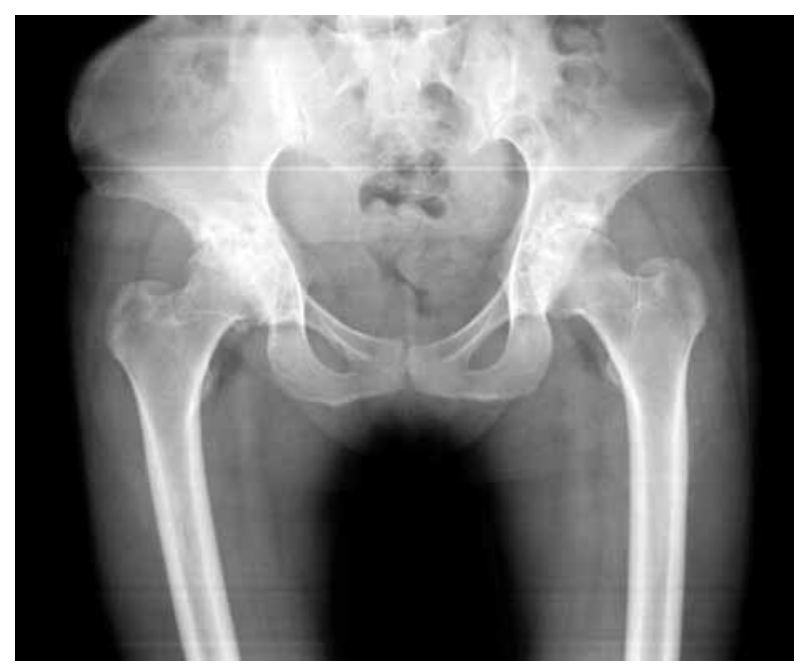

Figure 2. Typical osteoarthritic signs (joint space narrowing, subchondral sclerosis, and sclerotic cystic formations) is revealed on the pelvic radiograms of our patient. 
In the majority of cases, arthropathy is seen in advanced stages, but it can be the first sign in the iron accumulation process. ${ }^{6}$ However, it is unrelated to the iron load in the body. Involvement of the wrists along with the MCP (mainly the second and third digits), and PIP joints, which mimicks OA, are the most characteristic findings, but the preservation of the DIP joints is important in order to discriminate the arthropathy from primary OA. New, hook-like bone formations on the heads of the metacarpal bones are critical radiological signs that signal the presence of $\mathrm{HH}^{7-9}$ In our patient, the clinical and radiological characteristics of joint involvement supported our diagnosis of $\mathrm{HH}$.

Short-lived acute inflammatory episodes triggered by calcium pyrophosphate crystals can mimic RA,, 10 but our radiographic and laboratory findings ruled out this diagnosis. Testing for anti-CCP antibodies and RF is useful to help discriminate $\mathrm{HH}$ from RA. In our case, the conjunction of negative RF and anti-CCP tests made this diagnosis unlikely. Additionally, the ESR and CRP levels were normal, and the presence of characteristic radiological findings, including metacarpal osteophytes and subchondral sclerosis, was consistent with $\mathrm{HH}$ since those are usually not seen in patients with RA. Typical radiographic findings of RA include periarticular osteopenia and marginal erosions.

In patients with clinical and radiological findings that comply with $\mathrm{HH}$, biochemical (serum iron, ferritin, and transferrin saturation), and genetic hemochromatosis gene (HFE) mutation tests can establish the diagnosis. A transferrin saturation analysis is the most sensitive test to demonstrate iron load in the body, and Feder et al. ${ }^{11}$ determined that its cut-off value was $45 \% .{ }^{11}$ In our case, though serum iron and ferritin levels were within normal limits, the transferrin saturation rate was higher than normal (69.71\%).

Serum ferritin levels can increase as an acute phase reactant, yet potentially normal levels have been detected in younger $\mathrm{HH}$ patients. Thus, this test has a lower sensitivity. It also should not be forgotten that in $\mathrm{HH}$, serum iron levels and the transferrin saturation rate can be within normal limits in cases with an excessive accumulation of iron in reticuloendothelial system organs and higher ferritin levels. ${ }^{12}$
The HFE gene is located near the human leukocyte antigen locus on chromosome $6,{ }^{13}$ and more than $90 \%$ of the patients with this disease possess the $\mathrm{C} 282 \mathrm{Y}$ mutation of this gene. Moreover, homozygous and heterozygous genotypes correlate with major and minor disease expression, respectively. ${ }^{1,2}$ In recent years, another type of mutation (H63D) has been potentially linked to $\mathrm{HH}$, and this induces the substitution of aspartic acid in place of histidine on position 63 of the HFE gene. ${ }^{14}$ In cases with compound heterozygosity in which both mutations are found in combination (C282Y/H63D), a moderate degree of iron accumulation in the body has been observed. ${ }^{3}$ In our patient, the homozygous H63D mutation was discovered as a result of genetic examinations, but it is not as penetrant as the $\mathrm{C} 282 \mathrm{Y}$ mutation. In addition, there have been very few reported cases of hemochromatosis with this genotype. ${ }^{1}$

If C282Y homozygosity or C282Y/H63D compound heterozygosity without hepatomegaly and normal serum transaminase levels are detected, then a liver biopsy is not recommended, but when higher hepatic enzymes or hepatomegaly are present, this type of biopsy should be performed. ${ }^{15}$ In our patient, since the fasting serum ferritin and enzyme levels were within normal ranges without hepatomegaly, no liver biopsy was needed.

To demonstrate iron accumulation, instead of a liver biopsy, a noninvasive MRI technique using a superconducting quantum interference device can be utilized. ${ }^{2}$ Iron accumulation in tissues markedly shortens $T_{2}$ and $T_{2}$ relaxation times. Therefore, an MRI is a specific, sensitive method that can be used to detect iron overload. Normal liver tissue has a signal intensity brighter than that of skeletal muscles in all sequences; hence, skeletal muscle is used as a reference tissue for the evaluation of hepatic signals. In hemochromatosis, an MRI typically can identify decreased signal intensity in the liver and pancreas. ${ }^{16}$

It has also been reported that lower iron accumulation is better demonstrated in gradient echo sequences, whereas iron overload is better visualized in spin echo sequences. ${ }^{17-19}$ In our case, the hepatic iron load was estimated to be $44 \pm 20 \mu \mathrm{mol} / \mathrm{g}$ (normal $<36 \mu \mathrm{mol} / \mathrm{g}$ ) in $\mathrm{T}_{2}$ sequences, which is consistent with mild degrees of iron load. 


\section{Conclusion}

Hereditary hemochromatosis can be confused with other arthropathies, especially those that affect the hand joints. To diagnose $\mathrm{HH}$, a screening test to measure the transferrin saturation rate should be performed initially. Then, noninvasive supportive diagnostic methods, such as HFE gene analysis and an MRI for the detection of increased hepatic iron load should be utilized. Finally, if there is still suspicion, a definitive diagnosis can be made via a biopsy of the affected tissues.

\section{Declaration of conflicting interests}

The authors declared no conflicts of interest with respect to the authorship and/or publication of this article.

\section{Funding}

The authors received no financial support for the research and/or authorship of this article.

\section{REFERENCES}

1. Limdi JK, Crampton JR. Hereditary haemochromatosis. QJM 2004;97:315-24.

2. Pietrangelo A. Hereditary hemochromatosis--a new look at an old disease. N Engl J Med 2004;350:2383-97.

3. Tüzün Y, Yakut M. Demir metabolizması ve herediter hemokromatozis. Güncel Gastroenteroloji 2009;13:94-101.

4. Schumacher HR Jr. Hemochromatosis and arthritis. Arthritis Rheum 1964;7:41-50.

5. Schumacher HR. Articular cartilage in the degenerative arthropathy of hemochromatosis. Arthritis Rheum 1982;25:1460-8.

6. Faraawi R, Harth M, Kertesz A, Bell D. Arthritis in hemochromatosis. J Rheumatol 1993;20:448-52.

7. AxfordJS. Rheumaticmanifestations of haemochromatosis. Baillieres Clin Rheumatol 1991;5:351-65.

8. Carroll GJ, Breidahl WH, Bulsara MK, Olynyk JK. Hereditary hemochromatosis is characterized by a clinically definable arthropathy that correlates with iron load. Arthritis Rheum 2011;63:286-94.
9. Camaschella C, De Gobbi M, Roetto A. Hereditary hemochromatosis: Progress and perspectives. Rev Clin Exp Hematol 2000;4:302-21.

10. Njajou OT, Vaessen N, Joosse M, Berghuis B, van Dongen JW, Breuning MH, et al. A mutation in SLC11A3 is associated with autosomal dominant hemochromatosis. Nat Genet 2001;28:213-4.

11. Feder JN, Gnirke A, Thomas W, Tsuchihashi Z, Ruddy DA, Basava A, et al. A novel MHC class I-like gene is mutated in patients with hereditary haemochromatosis. Nat Genet 1996;13:399-408.

12. Moirand R, Jouanolle AM, Brissot P, Le Gall JY, David V, Deugnier Y. Phenotypic expression of HFE mutations: a French study of 1110 unrelated ironoverloaded patients and relatives. Gastroenterology 1999;116:372-7.

13. McDonnell SM, Preston BL, Jewell SA, Barton JC, Edwards CQ, Adams PC, et al. A survey of 2,851 patients with hemochromatosis: symptoms and response to treatment. Am J Med 1999;106:619-24.

14. Olson LJ, Edwards WD, Holmes DR Jr, Miller FA Jr, Nordstrom LA, Baldus WP. Endomyocardial biopsy in hemochromatosis: clinicopathologic correlates in six cases. J Am Coll Cardiol 1989;13:116-20.

15. Guyader D, Jacquelinet C, Moirand R, Turlin B, Mendler $\mathrm{MH}$, Chaperon J, et al. Noninvasive prediction of fibrosis in C282Y homozygous hemochromatosis. Gastroenterology 1998;115:929-36.

16. Semelka RC, Mitchell DG. Liver and biliary system. In: Edelman RR, Hesselink JR, Zlatkin MB, editors. Clinical magnetic resonance imaging. 2nd ed. Philadelphia: Saunders; 1996. p. 1490-2.

17. Guyader D, Gandon Y. Quantification of iron overload. Bull Acad Natl Med 2000;184:337-47.

18. Ernst O, Sergent G, Bonvarlet P, Canva-Delcambre V, Paris JC, L'Herminé C. Hepatic iron overload: diagnosis and quantification with MR imaging. AJR Am J Roentgenol 1997;168:1205-8.

19. Kreeftenberg HG Jr, Mooyaart EL, Huizenga JR, Sluiter WJ. Quantification of liver iron concentration with magnetic resonance imaging by combining T1-, T2-weighted spin echo sequences and a gradient echo sequence. Neth J Med 2000;56:133-7. 